\title{
AN OPEN PROBLEM ON BOUNDARY BEHAVIOUR OF HOLOMORPHIC MAPPINGS
}

\section{Ewa Ligocka (Warszawa)}

In the study of the boundary behaviour of proper holomorphic mappings between weakly pseudoconvex domains with $C^{\infty}$-boundaries the following property plays the fundamental role:

Property $R$. A domain $D$ has the property $R$ iff the Bergman projection $P: I^{2}(D) \rightarrow I^{2} H(D)$ is a continuous operator from $C^{\infty}(\bar{D})$ into itself with the usual Fréchet topology.

A sufficient (but not necessary) condition for the property $\mathrm{R}$ is the following: A smooth pseudoconvex domain has the property $\underline{\mathrm{R}}$ if for each $s>0$ there exists an operator solving the $\bar{\partial}$-problem, which is a compact operator $T_{s}$ from the space $W_{\langle 0,1\rangle}^{\mathrm{s}}$ of $\overline{\mathrm{o}}$-closed $\langle 0,1\rangle$-forms with the $s-$ th Sobolev norm into the Sobolev space $W^{5}(D)$.

The PROBLEM posed is to characterize the domains $D$ for which such compact operators exist. In particular, does there exist a domain $D$ for which the subelliptic estimates for the $\bar{\partial}$-Neumann problem are not valid and for which there exist compact operators which solve the $\bar{\partial}-$ problem? 